\title{
Primary care physicians' experience with family history: An exploratory qualitative study
}

\author{
Janet L. Williams, MS, CGC, ${ }^{l}$ Dave S. Collingridge, $P h D,{ }^{2}$ and Marc S. Williams, $M D^{l}$
}

\begin{abstract}
Purpose: Family history can guide patient care but is underused. Physician experience with family history has been inadequately characterized. The study's purpose was to assess primary care physicians' experiences with family history. Methods: A qualitative study using an existential-phenomenological approach. Primary care physicians using an electronic health record to enter family history participated in semistructured interviews. Themes were developed relating to physicians' experiences with collection and documentation of family history. A summary describing each physician's experience was developed and analyzed. The themes and experiences from each primary care physician were synthesized across all participants. Results: Positive and negative experiences were identified. Positive experience was associated with the perceived usefulness of family history to guide patient care, confidence using family history, practice efficiency, and enhancing the physician-patient relationship. Negative experience was primarily associated with perception that family history had to be collected and process problems, although confusion about the use of family history, perceived inaccuracies and incompleteness of the information provided, time, and potential liability contributed to negative experience. Most primary care physicians had an overall positive experience with family history, although the balance of the positive and negative experiences did not seem related to the degree the electronic health record was used to enter family history. Conclusions: The primary care physicians' experience with family history represents the synthesis of tensions between positive and negative experiences relating to collection and use. Understanding the components of the experience could inform redesign of systems to enhance the positive and reduce the negative elements. Genet Med 2011:13(1):21-25.
\end{abstract}

Key Words: family history, primary care, physician-patient relations, decision making, computerized medical record

F amily history $(\mathrm{FH})$ plays an important role in identifying disease risk and guiding diagnosis and preventive care.1,2 The Centers for Disease Control and Prevention have identified diseases where adequate evidence exists to stratify risk and modify care based on $\mathrm{FH} .{ }^{3}$ Despite this, physicians spend little time collecting FH. ${ }^{4}$ Articles have identified what FH is (or should be) collected by primary care physicians (PCPs; specific diseases, age of onset, relationship to patient, etc. $)^{4-6}$ and identified barriers to collection of FH (time, perception that information from patient is not accurate, or actionable). ${ }^{7}$ Other studies have indicated that many PCPs have concerns about their ability to use the FH they collect. ${ }^{8,9}$ Although each of these

From the ${ }^{1}$ Clinical Genetics Institute; and ${ }^{2}$ Statistical Data Center, Intermountain Healthcare, Salt Lake City, Utah.

Marc S. Williams, Clinical Genetics Institute, 324 10th Avenue, Suite 183, Salt Lake City, UT 84103. E-mail: marc.williams@imail.org.

Disclosure: The authors declare no conflict of interest

Submitted for publication April 27, 2010.

Accepted for publication August 24, 2010.

Published online ahead of print November 4, 2010.

DOI: $10.1097 /$ GIM.0b013e3181f928fc studies generated data regarding specific aspects of collection and use of FH, none have attempted to examine physicians' experience with obtaining and using a FH. It is reasonable to assume that overall experience may impact a physician's collection and use of FH. The purpose of this study is to assess PCPs' experiences with FH.

\section{METHODS}

\section{Study population and sampling strategy}

Intermountain Healthcare is an integrated health care delivery system that annually cares for $>1$ million patients in Utah and southern Idaho. An electronic health record (EHR) is available to all employed physicians and many affiliated physicians. FH can be entered in the EHR in three ways (through a webform, the problem list, and chart notes). A convenience sample of 25 PCPs affiliated with Intermountain Healthcare who have used the EHR in the past year to record FH on at least 10 patients and worked within 50 miles of our site (to allow face-to-face interviewing) were identified. We followed a purposive, maximum variation sampling strategy while identifying the 25 PCPs in that we selected PCPs with varying levels of experience with using the EHR to record FH and selected PCPs from various practice sizes and settings (i.e., family practice $[\mathrm{FP}]$, general internal medicine [IM], and pediatrics [P]). All 25 PCPs were invited to participate. Four declined at the outset. Only one of the 25 was female, and, while willing to participate, she was unable to be scheduled. Of the remaining 20 male PCPs, 16 were interviewed. PCP's age and years in practice were not obtained, although in the course of the interviews some information was obtained that indicated a range of time from approximately 1 year to $>30$ years in practice. All the PCPs with one exception spent $>75 \%$ of time in direct patient care. One pediatrician (P1) has a half-time administrative position. Approximately half of the PCPs practiced in large single or multispecialty groups in the Salt Lake Valley, whereas the others practiced in smaller suburban group practices. None of the PCPs had a large inpatient practice.

\section{Data collection}

We collected data with semistructured, interpersonal interviews. This interview format was followed because it was ideal for eliciting rich descriptions of experiential phenomena, gave participants the opportunity to elaborate on their responses, and enabled interviewers to immediately explore issues and gain clarity. The content and format (open ended) of the questions (Table 1) were created to facilitate meaningful descriptions of PCPs' experiences. Questions were constructed based on information obtained from prior studies of PCPs and $\mathrm{FH}^{4-9}$ with additional questions being developed to address areas of interest that had not been reported in these studies. The authors evaluated the potential of each question to elicit rich descriptions and made revisions, as needed. A physician group that routinely advises the Clinical Genetics Institute and is composed of PCPs and specialists was used to review the questions. Suggestions 


\section{Table 1 Interview questions}

1. Describe your current practices related to family history collection. (May need to ask prompting or clarifying questions depending on response. Examples might include use of forms, who enters the data, and how is it presented to physician. Some of these are also covered below. How do you avoid collecting the same information repeatedly? How often do you update the family history?)

2. At what type of visit do you collect family history information? How does the type of visit affect what type of information is collected?

3. How do you collect and record family history information in the medical record?

4. What family medical history information is most important to collect?

5. How do you use family history?

6. How do you interpret the family medical history?

7. What types of recommendations are impacted by family history?

8. How do you discuss this information with your patients? How do patients respond to this information?

9. Describe what you find useful about family medical history in your clinical care.

10. What barriers do you see or experience in using family history in clinical care?

11. What part of the EHR do you use to enter family history? Why? Have you tried other parts of the EHR for this purpose? What did you like and dislike?

12. What might be potential solutions to the barriers? Or improvements for the current medical record options.

13. If you could design a perfect family medical history vehicle, what do you envision? See as essential?

EHR, electronic health record.

were incorporated into the final question set. Interviews were performed by two of the authors (J.L.W. and M.S.W.). J.L.W. conducted six interviews, and M.S.W. conducted 10 interviews. J.L.W. and M.S.W. discussed the interview process and used the same questions (Table 1) to maximize interinterviewer reliability.

During interviews, question order varied depending on responses from PCPs. Interviews lasted anywhere from 30 to 60 minutes. This time frame was sufficient to gather meaningful descriptions of collecting FH. In five instances where we did not gather sufficiently meaningful descriptions because the PCPs focused on technical issues and questions related to $\mathrm{FH}$ rather than relating experience about $\mathrm{FH}$, the interview data were excluded from analysis. Three of these PCPs were interviewed by J.L.W. and two by M.S.W. This study was approved by the institutional review and privacy boards of Intermountain Healthcare.

\section{Data analysis}

The interviews were transcribed, and analysis was carried out using the existential-phenomenological approach in the tradition of Edmund Husserl ${ }^{10}$ following the method outlined by Polkinghorne. ${ }^{11}$ (Readers desiring a thorough description of this approach may refer to Polkinghorne ${ }^{11}$ and Collingridge. ${ }^{12}$ ) Ex- istential phenomenology combines Soren Kierkegaard's existential philosophy with Edmund Husserl's phenomenological approach to understanding the world. According to this combined approach, truth is experiential, or in other words, truth is given in personal experience. With personal experience as its main subject matter, the purpose of existential phenomenology is to capture descriptive accounts of experiential phenomena, to understand phenomena as they are experienced by the participants.

There were some minor adjustments to the method as a consequence of three people being involved in the data analysis, but the main components of the analysis remained the same. In the first step, J.L.W. and M.S.W. read through all the transcripts to get a sense of the whole. This provided a cursory understanding of what it is like for PCPs to collect and use FH information. D.S.C., an expert on existential phenomenology, joined J.L.W. and M.S.W. for the remainder of the analysis.

Second, each researcher read through the transcripts and while doing so, located and identified meaning units. Locating meaning units involves locating statements that represent selfcontained expressions relevant to the experience under investigation. Identifying meaning units involves carefully reflecting on what was said to move beyond the linguistic expression to the reality being described in each unit.

Third, the researchers met multiple times to discuss each transcript and the meaning units identified. In their discussions, they further reflected on the essence of the meaning units and used imaginative variation to confirm their identities. Imaginative variation involves slightly altering different aspects of a participant's description of an experience to better grasp its essential meaning. Asking "What if ...?" and "Would things be different if ...?" questions of the PCPs' experiences resulted in greater confidence regarding the accuracy of the meaning units identified.

Fourth, the research team related meaning units to other meaning units within the same transcripts and created descriptive summaries of each PCP's experience with collecting FH. Specifically, descriptive summaries were created by identifying patterns in meaning units for each participant and reducing these into context-specific descriptions of what it is like for each $\mathrm{PCP}$ to collect FH.

Finally, the research team took the individual summaries created in Step 4 and, through common consensus, merged them into themes. The themes were then synthesized into a general description of the participants' experience with collecting FH. How the themes varied according to situational and participant characteristics was also explored. The result was a rich and varied description of PCPs' experiences with FH. Each member was in agreement on the final emergent themes, general description, and demographically derived comparisons.

Each interview was subsequently classified by the group as an overall positive or negative experience based on subjective (e.g., intensity of language) and objective (e.g., counts of meaning units) indicators identified by each of the authors across the positive and negative experience domains. There was general agreement between researchers on the classification.

\section{RESULTS}

Five interviews were excluded because the interviewees did not relate experientially meaningful data (focus on technical issues). The specialties of the 11 PCPs analyzed were FP, 5; IM, 4 ; and P, 2. Contributors to positive and negative experience are summarized in Table 2 . 
Table 2 Summary of themes relating to positive and negative experience with $\mathrm{FH}$ collection

\begin{tabular}{ll}
\hline Experience & \multicolumn{1}{c}{ Theme } \\
\hline Positive & Perceived usefulness of FH to guide patient care \\
& Enhances the doctor/patient relationship \\
& Confidence using FH \\
& Improves practice efficiency \\
& $\begin{array}{l}\text { Perceived requirement to collect FH irrespective } \\
\text { of usefulness } \\
\text { Challenges with the process of collecting and } \\
\text { recording FH including time constraints } \\
\text { Confusion about the use of FH including lack of } \\
\text { guidelines } \\
\text { Issues of perceived inaccuracy and incompleteness } \\
\text { of patient provided FH information } \\
\text { Exposure to potential liability }\end{array}$ \\
\hline FH, family history. &
\end{tabular}

Positive experiences were related to the perceived usefulness (utility) of the information of which there were two components: directing patient care based on $\mathrm{FH}$ and building relationship/rapport. Regarding the former, a pediatrician stated, "[W]hat I am looking for are things that could possibly play a role in the 18-22 years that I am seeing that patient." (P1). Another said, "I am ... focusing in on the major killers of my patients ... those kind of things we have some good interventions [for] or if we find them early enough we can help prevent major complications." (FP1). Although most PCPs did not relate experiences regarding rapport, those who did invariably described positive experiences. A pediatrician stated, "I certainly know more about the family and how to relate to them ..." (P2). A family physician stated, "One of my biggest joys in that interview is finding out about the individual ... - how they grew up, what influences they had ... finding out about someone who grew up with a chronically ill ... sibling can tell you a lot more about that individual ..." (FP2). The PCP's attitude could impact rapport. When asked whether patients bring back requested FH information, an internist said, "... it depends on how worried I make them." (IM1).

Two pediatricians held very positive attitudes toward FH. One related no negative experience with $\mathrm{FH}$, whereas the other expressed some process issues that were outweighed by the perceived value of the information and the downstream impact on time. "I could see some people saying time would be a barrier, but I think ... the time I take to collect and manage this saves me time so much down the road ... I have taken minutes and now my subsequent visits are seconds that you spend with them. I don't think time should be a barrier. In terms of stratification most of it is down the road stuff. It is tucked away for when that child has a symptom that might ... prompt me to act a little bit sooner." (P1).

Negative experiences related to two issues: perception that FH had to be collected and process problems. The perception that one is expected to collect $\mathrm{FH}$ was generally attributed to external sources. "We are hit constantly with, "here are the new recommendations for this and for that' and so you are constantly updating in your mind when you are going to be doing different things.” (FP3). In contrast, an internist's experience indicated that the expectations arose from the practitioner himself. "I just kind of choose to get a little bit more history so that I can note exactly what it means. ... They [paper intake forms] are filled out for every new patient and every annual physical. That is how I do it. Some people don't do it for the physicals; they only do it for the new patients." (IM1). Most interviewees related negative experience with the process of collecting or entering FH information. An internist complained about the webform (the electronic form to enter FH), "No, it is useless. That is a big problem. ... It is impossible to read or navigate when you go back and look at it." (IM1). Commenting on the transition from a paper-based to paperless system, another internist stated, "I have taken these meticulous records and now I don't have them any more. There is no place to add anything else." (IM2). The PCP who expressed the most frustration related no positive experience and expressed negative experiences with virtually every aspect of FH. He also expressed skepticism about the accuracy or usefulness of the information. "The patient's understanding of what the disease process was is often limited and we are not going to get to it." (IM3).

Other less common but meaningful sources of negative experience were identified. Several interviewees expressed concern about potential liability if FH is collected but not acted on, and the patient experienced a potentially preventable outcome. A family physician stated: "When it became possible for me to have all this information in one place then I started to develop all this liability to go through it." (FP4). The amount of information can also create a negative experience as expressed by an internist "[Y]ou end up with like $10+$ pages of yes, no, yes, no and it is impossible to read or navigate when you go back and look at it." (IM1).

Another important finding concerned the PCP's confidence in using the information collected to help the patient. PCPs who expressed confidence in using $\mathrm{FH}$ related more positive experiences around the information than those who were not as certain about how to use it. When an internist was asked what information he collects, he replied "I would love to know this person has a family history of colon cancer. ... To have that sit right there as family history of colon cancer and see no colonoscopy, that to me is another way of making sure something doesn't fall through the cracks." (IM2). A pediatrician noted, "If I am seeing a two-year-old and they have now come in with a third episode of wheezing and there is a history of atopy in the family and I say, 'you know what, clinically your child has asthma. ... Let's get him on a controller'.” (P1). In contrast, a family practitioner related, "I am still not always sure what to do with the information once I have it. ... How does this help me take care of my patients?" (FP3). This uncertainty even expressed itself as guilt in one internist: "[I]t is still embarrassing to me why I occasionally have a person who has never had a colonoscopy and they have colon cancer in their family." (IM2).

Most frustration stemmed from collecting and entering FH in the EHR. Many PCPs create a patient worksheet to organize the visit. The FH webform must be translated by computer into the worksheet, and all positives and negatives are printed, resulting in pages of single-spaced text. An internist noted, “... it [FH] shows up down at the bottom of this big jumble of stuff that nobody ever looks at." (IM1). In contrast, the problem list appears as a short list. One PCP stated, "That [the problem list] is the only place I found to flag it and have it be readily available" (FP5), whereas another said, "I will probably double enter family history [in the Webform and the problem list] because the webform is not that practical for me ..." (FP4). Although most interviewees related negative experiences regarding the process of collecting and entering $\mathrm{FH}$, some shared 
positive experiences. One pediatrician related a unique perspective, "[The time I take to collect and manage this saves me time so much down the road." (P1). This is an interesting perspective as the same individual stated, "The reality is the information $99 \%$ of the time doesn't come into play in your experience." He summarized his thoughts as follows: "I think the value that is there from a business standpoint and healthy practice standpoint could be underscored with physicians as you go forward." Although others noted potential enhancement of reimbursement, the value expressed in this statement reflects not only reimbursement but also an increase in practice efficiency. This physician is a part-time administrator within the Intermountain system raising the question of whether this position confers a perspective not reflected in other interviews.

One PCP (IM3) had a completely negative experience with FH collection. Two interviews were classified as ambivalent to slightly positive (FP3 and IM1). To determine whether there was a relationship between experience and use of the EHR to enter FH, participant usage was assessed. As we did not have access to medical records for this study, we were unable to determine what percentage of a given PCP's patients had FH entered in the EHR although numbers of unique patients entered was determined. Usage was defined as the number of unique patients with $\mathrm{FH}$ data entered by the participant in the previous 2 years. Informal review of the data for the 16 participants revealed that a cutoff of 100 patients entered evenly divided participants into two groups defined as high users (more than 100 patients entered) and low users (fewer than 100 patients entered). After exclusion of the five noninformative interviews, of the remaining 11 interviews, seven were high users and four were low users. No relationship was noted between the balance of the positive and negative experiences and the degree of usage. The PCP who reported an overall negative experience (IM3) and a PCP who expressed a barely positive experience history (IM1) were high users of the system. Of the two PCPs with the highest positive experience with $\mathrm{FH}$, one was a high user (P1) and the other a low user (P2). Five PCPs expressed positive experience with both the utility domains, but four of the five were low users.

\section{DISCUSSION}

A tension exists between the perceived positive and negative experiences with FH. Positive experiences were most often associated with impact on patient care (utility) and enhancing the physician-patient relationship. Negative experiences resulted from the expectation to collect $\mathrm{FH}$ and the laborious process. Frustration was directly related to the perceived negative experience. Other studies identified similar themes, ${ }^{13,14}$ although the studies are not directly comparable as the first study focused on the reaction to a "genetic family history" and the second the "cancer family history." Wood et al. ${ }^{14}$ specifically noted the positive role of FH in building relationship and rapport something that has received less attention in the $\mathrm{FH}$ literature. Although encounter time has been identified repeatedly as a barrier to collection of FH, the concept of time as an investment to improve care and future efficiency has not been reflected in other studies.

PCP confidence using FH also relates to positive experience. This finding is supported by a study that examined how physicians choose which clinical questions to pursue. ${ }^{15}$ Although this study did not focus on FH per se, the authors found that the single best predictor of physician attention to a question was the physician's belief that a definitive answer existed. The impor- tance of confidence that we found in our study has been highlighted in other studies. , $^{, 9,14}$

Problems with information overload that we discovered have been seen in other studies. Physicians only address approximately $30 \%$ of the clinical questions posed in a typical encounter. ${ }^{15}$ Some of our interviewees perceived that collecting more FH information than can be acted on creates liability concerns. However, the previous study did not identify this concern. ${ }^{15}$ Additionally, a 1999 review of liability relating to failure to identify risk for cancer found that many of the claims resulted from not inquiring about a $\mathrm{FH}$ of cancer rather than failing to act on FH information that was collected. ${ }^{16}$ More study of the impact of liability is warranted. PCPs in another study expressed the desire for more guidelines around cancer FH. ${ }^{14}$ Guidelines are useful to condense information into clinically actionable recommendations. As noted by those interviewed in the referenced study, few guidelines for using FH exist. PCPs in our study discussed $\mathrm{FH}$ guidelines that they use in practice but did not indicate that more guidelines were needed. The need for more FH guidelines should be explored.

Another component of positive experience is the role $\mathrm{FH}$ collection plays in building relationships. This was mentioned by some respondents in a previous study limited to cancer $\mathrm{FH},{ }^{14}$ but based on our results, it seems to be relevant for general $\mathrm{FH}$ collection as well. The paucity of information on the benefits of relationship building is surprising given our findings and the results of a recent study by Hall et al. ${ }^{17}$ Although examining PCP and patient understanding and communication around FH of heart disease, it was noted that patients expressed disappointment if there was a mismatch between how they thought the PCP should respond to their FH and the actual PCP response. In this electronic era, although having patients enter their $\mathrm{FH}$ electronically (outside of the encounter) may improve clinical efficiency, separating the collection of $\mathrm{FH}$ from the encounter could adversely impact rapport building. This concern was shared by PCPs using paper-based FH collection tools. ${ }^{14}$ However, if FH risk is stratified and combined with patient input about their FH concerns, it could focus the visit to not only improve utility but also enhance rapport building. Patient and provider experience around rapport and relationship associated with FH collection is an area deserving of additional study.

Surprisingly, some providers chose to increase their work by duplicating FH information in the webform and problem list to enhance utility. This illustrates the concept of the "workaround" where end users manipulate the system in ways unanticipated by designers to improve workflow. Using the problem list to enter FH is novel and could lead to improvements in the EHR, although an internist noted limitations with this approach, "If you get more than 10-15 items on the problem list it's hard to manage ..." (IM4).

We were able to compare the overall experience with $\mathrm{FH}$ and the objective usage for each interviewee. No relationship between high or low users and the balance of positive and negative aspects of their experience was found. The only interviewee who related a completely negative experience was the highest user having entered more than 1500 separate "Family history of ..." problems in 1100 unique patients. This suggests that usage of FH in EHR is influenced by more than just experience.

This study has several limitations. All participants were known to enter FH in the EHR. This excluded PCPs who collect FH but are not using the EHR to record it, and thus, the findings of this study may not be generalizable to PCPs who do not enter FH into the EHR. A substantial contributor to negative experience was frustration entering FH into the EHR. The experience of PCPs who do not use the EHR for this purpose could be 
different. Also, all participants were male. There may be gender differences in the perceived experience around $\mathrm{FH}$, given that positive experience relates in part to developing rapport. A recent study finds that on attributes beyond technical skills that underpin a good doctor-patient relationship, women usually excel over men. ${ }^{18}$ There were differences between the two interviewers (M.S.W. is an MD medical geneticist and male, whereas J.L.W. is a certified genetic counselor and female). These physical, professional and stylistic differences could have impacted the responses. M.S.W. and J.L.W. have extensive experience with FH in clinical practice which could have introduced bias. The use of imaginative variation and inclusion of a researcher with expertise in existential phenomenology and no prior experience with $\mathrm{FH}$ collection or use helped mitigate these potential biases. Finally, although the current qualitative study is methodologically rigorous for its stated purpose, it is possible that surveying larger numbers of PCPs could generate additional themes that were not identified in this study.

In conclusion, we found that the experience of PCPs with FH represents the synthesis of tensions between positive experiences relating to utility and building rapport, and negative experiences relating to process problems and the perception that FH must be collected. A potential next step to extend these findings could be the construction of a validated questionnaire using the results of this study to be distributed to a larger number of male and female PCPs. Studies exploring the role of $\mathrm{FH}$ in the provider-patient relationship and factors that affect FH entry in the EHR are needed. Studies of this nature will further define the components of the experience, which could inform redesign of $\mathrm{FH}$ collection systems to enhance the positive and reduce the negative elements. Although such studies may provide information to improve the PCP's experience with $\mathrm{FH}$, this information may not impact the use of the EHR to document this information, as this seems to operate on principles other than experience which await characterization.

\section{ACKNOWLEDGMENTS}

This work was supported by Genetic Alliance Project ID No. 1002481 (HRSA funded) entitled "Utilizing Family Traditions and Oral History for Health Promotion." The authors thank Cami Bills for transcribing all the interviews and the study participants who gave generously of their time.

\section{REFERENCES}

1. Harris EL, McMullen C. Final report: family history in clinical practice, 2006. Available at: http://www.oregon.gov/DHS/ph/genetics/docs/Family_History. pdf. Accessed August 17, 2009.

2. Butterworth A, Pharoah P, 2007. Family history as a risk factor for common, complex disease. Available at: http://www.phgfoundation.org/pages/family_ history.htm. Accessed August 17, 2009.

3. Yoon PW, Scheuner MT, Jorgensen C, Khoury MJ. Developing Family Healthware, a family history screening tool to prevent common chronic diseases. Prev Chronic Dis 2009;6:A33.

4. Acheson LS, Wiesner GL, Zyzanski SJ, Goodwin MA, Stange KC. Family history-taking in community family practice: implications for genetic screening. Genet Med 2000;2:180-185.

5. Summerton N, Garrood PV. The family history in family practice: a questionnaire study. Fam Pract 1997;14:285-288.

6. Reid GT, Walter FM, Brisbane JM, Emery JD. Family history questionnaires designed for clinical use: a systematic review. Public Health Genomics 2009; $12: 73-83$.

7. Rich EC, Burke W, Heaton CJ, et al. Reconsidering the family history in primary care. J Gen Intern Med 2004;19:273-280.

8. Emery J, Watson E, Rose P, Andermann A. A systematic review of the literature exploring the role of primary care in genetic services. Fam Pract 1999; 16:426-445.

9. Gramling R, Nash J, Siren K, Eaton C, Culpepper L. Family physician self-efficacy with screening for inherited cancer risk. Ann Fam Med 2004; $2: 130-132$.

10. Husserl E. An introduction to existential-phenomenological thought in psychology. In: Valle RS, Halling S, editors. Existential-phenomenological perspective in psychology. New York: Plenum Press, 1989:3-16.

11. Polkinghorne D. Phenomenological research methods. In: Valle RS, Halling $\mathrm{S}$, editors. Existential-phenomenological perspective in psychology. New York: Plenum Press, 1989:41-60.

12. Collingridge DS. Phenomenological insight on being hindered from fulfilling one's primary responsibility to educate students. Alberta J Educ Res 2008;54:112-123.

13. Watson EK, Shickle D, Qureshi N, Austoker J. The 'new genetics' and primary care: GP's views on their role and their educational needs. Fam Pract 1999;16:420-425.

14. Wood ME, Stockdale A, Flynn BS. Interviews with primary care physicians regarding taking and interpreting the cancer family history. Fam Pract 2008;25:334-340.

15. Gorman PN, Helfand M. Information seeking in primary care: how physicians choose which clinical questions to pursue and which to leave unanswered. Med Decis Making 1995;15:113-119.

16. Severin MJ. Genetic susceptibility for specific cancers. Medical liability of the clinician. Cancer 1999;86:2564-2569.

17. Hall R, Saukko PM, Evans PH, Qureshi N, Humphries SE. Assessing family history of heart disease in primary care consultations: a qualitative study. Fam Pract 2007;24:435-442.

18. Firth-Cozens J. Doctors with difficulties: why so few women? Postgrad Med $J$ 2008;84:318-320. 